\title{
Ambientes de calidad en la infancia temprana
}

\author{
Paz Valverde-Forttes ${ }^{*}$
}

\footnotetext{
Doctora en Educación, Universidad de Montreal. Profesora, Facultad de Educación, Universidad de los Andes, Santiago de Chile, Chile.

Correo electrónico: pazvalverdeforttes@gmail.com
}

Recibido: 10 de octubre del 2014 Aprobado: 4 de noviembre del 2015

Cómo citar este artículo: Valverde-Forttes, P. (2015). Ambientes de calidad en la infancia temprana. Pensando Psicología, 11(18), 141-151. doi: http://dx.doi.org/10.16925/ pe.v11i18.1224

\section{Resumen}

Propósito: el artículo tiene por objetivo compartir parte de la experiencia de Chile en relación con la búsqueda y construcción de ambientes de calidad que favorezcan el aprendizaje y desarrollo de los niños entre cero y seis años. Tema: la promoción de ambientes de calidad, desde la intencionalidad pedagógica del educador, al organizar el tiempo, el espacio y las interacciones, promoviendo el aprendizaje de los niños y las niñas. Desarrollo: se trata de una exposición principalmente descriptiva del contexto chileno y de los resultados obtenidos frente a las evaluaciones disponibles a partir de la aplicación de dos instrumentos: ECERS-R e ITERS-R y CLASS, en el marco de la implementación de programas que buscan mejorar la calidad, tales como Quik y Un buen comienzo de la Fundación Oportunidad. Conclusiones: a la luz de estos resultados, se releva la importancia del tema y se realiza un análisis de las políticas públicas emergentes y los desafíos o las tensiones que siguen presentes. Entre ellas, se destaca la tensión entre calidad y cobertura, así como la problemática de las desconfianzas entre los actores involucrados frente al significado y a la realidad de una educación de calidad.

Palabras clave: aprendizaje, desarrollo infantil, evaluación. 


\title{
Quality Environments in Early Childhood
}

\begin{abstract}
Purpose: The objective of the article is to share some of the experience of Chile in relation to the search for and creation of quality environments that would favor learning and development among children between zero and six years of age. Topic: The promotion of quality environments based on the pedagogical intention of educators, to organize time, space and interactions, promoting learning among boys and girls. Development: This involves a mainly descriptive exposition of the Chilean context and other results obtained through the available evaluations based on the application of two instruments: ECERS-R and ITERS-R, and CLASS, in the framework of the implementation of programs aimed at improving quality, such as Quik and Un buen comienzo of Fundación Oportunidad. Conclusions: These results demonstrate the importance of the topic and an analysis is made of emerging public policies and the challenges or tensions that persist. These include the tension between quality and coverage, along with the problem of distrust among the actors involved about the significance and reality of a quality education
\end{abstract}

Keywords: learning, childhood development, evaluation.

\section{Ambientes de qualidade na primeira infância}

\section{Resumo}

Propósito: este artigo tem como objetivo compartilhar parte da experiência do Chile sobre a busca e a construção de ambientes de qualidade que favoreçam a aprendizagem e o desenvolvimento das crianças entre zero e seis anos. Tema: a promoção de ambientes de qualidade, a partir da intencionalidade pedagógica do educador, ao organizar o tempo, o espaço e as interações, promovendo a aprendizagem das crianças. Desenvolvimento: trata-se de uma exposição principalmente descritiva do contexto chileno e dos resultados obtidos de avaliações disponíveis a partir da aplicação de dois instrumentos: ECERS-R e ITERS-R e CLASS, no âmbito da implementação de programas que buscam melhorar a qualidade, tais como Quik e Un Buen Comienzo, da Fundación Oportunidad. Conclusões: à luz desses resultados, revela-se a importância do tema e realiza-se uma análise das políticas públicas emergentes e dos desafios ou tensões que continuam presentes. Entre elas, destaca-se a tensão entre qualidade e cobertura, bem como a problemática das desconfianças entre os atores envolvidos diante do significado e da realidade de uma educação de qualidade.

Palavras-chave: aprendizagem, desenvolvimento infantil, avaliação. 


\section{Concepto de calidad}

El niño está hecho de cien. El niño tiene cien lenguas, cien manos cien pensamientos, cien maneras de pensar, de jugar y de hablar, cien siempre cien maneras de escuchar, de sorprenderse de amar, cien alegrías para cantar $y$ entender, cien mundos que descubrir, cien mundos que inventar, cien mundos que soñar.

El niño tiene cien lenguas, pero le roban noventa y nueve. La escuela y la cultura le separan la cabeza del cuerpo. Le dicen: de pensar sin manos, de actuar sin cabeza, de escuchar y no hablar, de entender sin alegría, de amar y sorprenderse sólo en Pascua y en Navidad. Le dicen: que descubra el mundo que ya existe y de cien le roban noventa y nueve. Le dicen: que el juego y el trabajo, la realidad y la fantasía, la ciencia y la imaginación, el cielo y la tierra, la razón y el sueño, son cosas que no van juntas. Y le dicen que el cien no existe. El niño dice: "en cambio el cien existe".

Loris Malaguzzi, 1996

Aguerrondo (1993) explicita que se ha hecho una definición restrictiva del concepto de calidad, lo que expresa una visión limitada del problema. La autora plantea la importancia de considerar que el concepto de calidad:

- Es complejo y totalizante: se trata de un concepto multidimensional, lo cual permite que sea aplicado a cualquiera de los elementos que entran en el campo de lo educativo.

- Está social e históricamente determinado: la calidad se lee de acuerdo con patrones históricos y culturales que tienen que ver con una realidad específica, en un lugar y momento determinado.

- Se constituye en imagen-objetivo de la transformación educativa: orienta la toma de decisiones. De ahí la importancia de explicitar qué se entiende por calidad en cualquier proceso de reforma.

- Se constituye en patrón de control de la eficacia del servicio: entendiendo que un sistema educativo eficiente no es aquél que tenga menos costo por alumno, sino aquel que optimizando los medios de los que dispone, sea capaz de brindar educación de calidad a toda la población.

Es a partir de las complejidades que emanan desde el mismo concepto de calidad que se sientan las bases de una temática, la cual ha motivado la búsqueda de respuestas frente a numerosas preguntas, tanto desde la teoría como desde la práctica, tanto para educadores en aula como para investigadores de distintos centros de desarrollo docente.

\section{La evaluación de la calidad en el nivel de educación parvularia}

\section{La educación parvularia y su impacto en el aprendizaje y desarrollo de los niños}

Según Egido (2000), se considerará la educación inicial como el período de cuidado y educación de los niños en los primeros años de su vida, aquella que se produce fuera del ámbito familiar. Esta concepción permite tener en cuenta las diversas modalidades educativas establecidas para niños desde el nacimiento hasta los cinco o seis años de edad.

A nivel mundial, esta etapa comprende una gran diversidad de prácticas, tales como guarderías, jardines infantiles, programas asistenciales, clases de preprimaria y patios de juego, entre otras. Estas prácticas pueden ser caracterizadas dentro de un continuo que comprende, en uno de sus polos un fin "asistencial", y en el otro, un fin "educativo". De esta manera, se podrían ubicar las distintas iniciativas que se implementan en esta etapa educativa de acuerdo con las necesidades detectadas, los objetivos que se proponen y las estrategias que se utilizan para lograrlos. Esta conceptualización puede visualizarse en la figura 1.

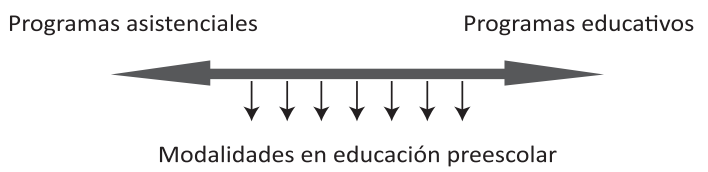

Figura 1. Polos en la definición de educación preescolar. Elaboración propia.

Las primeras manifestaciones de cuidado infantil institucionalizado y creado a partir del Estado, surgen como respuesta a crisis sociales que evidencian el abandono infantil (revolución industrial, posguerra, desastres naturales, pobreza). Estos servicios asistenciales se ubican en el extremo izquierdo de la figura 1. Los avances de la ciencia (psicología, sociología, neurociencia), y el trabajo de los precursores de la educación preescolar como Froebel, 
Decroly y Montessori, han consolidado la importancia de darle un carácter educativo a la atención del niño preescolar.

Autores como Clark-Stewart (1993), Goleman y Pence (1987), Howes (1988), así como Kontos y Fienes (1987) entre otros, determinaron que no siempre la asistencia a un centro de educación parvularia tenía efectos positivos frente al desarrollo y aprendizaje de los niños. Para que esto suceda, deben considerarse el nivel de calidad de dicho centro educativo: mayor calidad, efectos positivos; mala calidad, efecto negativo y no neutro. Una excepción la constituyen aquellas intervenciones en medios de riesgo psicosocial, en las que pareciera que sin tratarse de un centro con alto nivel de calidad, de todas maneras los niños se ven beneficiados en relación con su contexto: "Una escolarización iniciada tempranamente puede contribuir a la igualdad de oportunidades al ayudar a superar los obstáculos iniciales de la pobreza o de un entorno social y cultural desfavorecido" (Delors, 1996, p. 133).

Durante la década de los ochenta se llevaron a cabo una serie de estudios que tenían como objetivo evaluar la calidad de la educación de los primeros años. Junto con estas investigaciones, también se desarrollaron distintos programas de intervención y sus respectivos seguimientos, iniciándose así una línea de investigación sobre la educación infantil (LeraRodríguez, 2007).

De este modo, se pueden identificar dos grupos de variables a observar: las variables políticamente regulables o dimensión estructural, y las variables más ligadas al proceso educativo o a la calidad del contexto y sus relaciones. En sus inicios, las variables estructurales de la calidad se caracterizaban como aspectos de la educación inicial que estaban regulados (Phillips y Howers, 1987). Sin embargo, esta definición se ha expandido a fin de incluir variables tales como remuneraciones del personal, rotación de los educadores y mensualidad que pagan los padres, las cuales no están usualmente reguladas (Phillipsen, Burchinal, Howes y Cryer, 1997).

En esta oportunidad esta indagación se centra en las variables ligadas al proceso educativo y las interacciones, entendiendo que un programa educativo de calidad enfatiza prácticas que son asumidas para estimular el lenguaje, las capacidades intelectuales y físicas, la competencia social -incluyendo un balance de independencia y cooperación-, así como también el bienestar emocional. Además, se caracteriza por un enfoque centrado en el niño/a, enfatizando los juegos y las interacciones con materiales y otros niños/as, como los medios primarios que permiten obtener metas con relación al desarrollo. Esto requiere de un ambiente seguro y saludable. En esta definición, el rol del adulto es actuar como un facilitador de juegos que enriquecen a los niños/as y proporcionar atención, información nueva, recursos, apoyo y guía. En la práctica, esto significa que los adultos en los programas de alta calidad están atentos a los niños/as, a proporcionarles guía, demostrarles interés y ofrecerles amplias oportunidades para el aprendizaje, teniendo siempre como requisito el conocerlos individualmente y en profundidad.

\section{Ambiente de calidad: la organización del espacio y del tiempo}

La organización del espacio se presenta como la primera herramienta que tiene el educador para intencionar los aprendizajes de quienes habitarán dicho espacio. Si pensamos que los espacios reflejan las características de quienes lo habitan, el salón de actividades no es la excepción, ya que al entrar en él y observar lo que contiene y cómo se distribuye, es posible conocer algo de la identidad propia de ese educador y de sus estudiantes (Copple y Bredekamp, 2009).

Este espacio debe brindar distintas oportunidades de aprendizaje, debe permitir que los niños tengan la posibilidad de trabajar como grupo grande, en grupos pequeños e individualmente. Debe también estar ordenado a fin de permitir a los niños desenvolverse en forma autónoma dentro de él, accediendo a los elementos que necesiten para llevar a cabo su plan. No se debe olvidar que el uso de las paredes es un recurso potencial rico en oportunidades que permite a los niños interactuar en forma grupal o individual. Paredes, mobiliario y materiales, distribuidos en interiores y exteriores, deben constituirse como espacios seguros y acogedores, aportando de esta manera en la creación de un clima facilitador del aprendizaje.

Los criterios para definir las distintas alternativas de la organización del espacio, emanan de las propias características de los niños. Por ejemplo, si se sabe que los niños aprenden mejor a través del juego, la exploración y la actividad física, el educador debería usar muebles que se puedan mover, con el fin de crear espacios amplios, usar el mínimo de sillas y mesas. Deben evitarse pasillos que inviten a correr.

La organización de la rutina diaria es la segunda herramienta disponible para el educador. Qué hacer, en qué momento y por cuánto tiempo, son decisiones 
pedagógicas fundamentales para la creación de un ambiente de calidad. Una buena organización del horario ofrece una gama de diferentes tipos de actividades (Lavanchy, 1993) incluyendo:

- Tiempos activos y pasivos.

- Actividades de grupo pequeño y grande, así como tiempo para jugar solo y con otros.

- Tiempos de juego al interior y exterior.

- Un equilibrio de actividades para grandes y pequeños músculos.

- Tiempo para que los niños inicien sus propias actividades y tiempo para actividades iniciadas por el educador.

$\mathrm{Al}$ igual que frente a las decisiones que se toman respecto a la organización del espacio, la organización del tiempo debe responder a las características y necesidades de los niños. Por ejemplo, si sabemos que los niños tienen períodos de concentración aun limitados por lo que aprenden mejor en pequeñas dosis, los espacios de tiempo destinados al trabajo no podrán extenderse innecesariamente.

\section{Ambiente de calidad: las interacciones}

Una de las condiciones reconocidas en la literatura desde hace más de 20 años es que las interacciones que se producen entre educadores y niños son determinantes en la calidad de la acción educativa (Melhuish, 1993; Hadred y Sylva, 1994; Lavanchy, 1993; National Scientific Council on the Developing Child, 2004; Dombro, Jablon y Stetson, 2011). Estas interacciones deben reflejar una comunicación efectiva, cálida y respondiente.

El lenguaje que utilice el educador será determinante para el aprendizaje del niño y la comprensión que este construya del mundo que lo rodea (Meece y Soderman, 2010). Este lenguaje debe comprender preguntas significativas y abiertas, las cuales abran caminos para que el niño aprenda y se comunique. Se trata de un educador que responde a través de sus verbalizaciones en forma rápida y significativa a las necesidades e intereses de los niños, adaptándose a sus características individuales y al contexto cultural en que se encuentra.

Dentro de las interacciones que debe intencionar el educador, se encuentran también aquellas que llevan al niño a resolver sus conflictos, sobre todo considerando que se trata de una etapa caracterizada por el egocentrismo, lo cual facilita posibles dificultades en el contacto cotidiano con sus pares. Para esto, es necesario que los límites y las reglas sean claros, acordados previamente por todo el grupo (De Vries y Zan, 2003), y las estrategias de mediación bien orientadas y practicadas en forma coherente por los adultos que interactúan con los niños. Cautelar la instalación de este tipo de interacciones facilita el desarrollo de una mejor autoestima, mayor seguridad-apego y autonomía en el niño, lo que permite contar con las bases para el logro de un mayor y mejor aprendizaje.

Son los planes y la organización de clase que hace el maestro, su sensibilidad y capacidad de respuesta a todos los niños, $y$ sus interacciones con ellos en todo momento los que ejercen la mayor influencia sobre el desarrollo y el aprendizaje de los niños.

(NAEYC, 2009, p. 9)

\section{Algunos instrumentos que pueden evaluar calidad en el nivel inicial}

Luego de haber revisado brevemente las características de estas variables ligadas al proceso educativo, cabe presentar dos instrumentos que buscan evaluar calidad e incluyen las variables antes mencionadas.

\section{La Escala ECERS e ITERS}

La Escala de Calificación del Ambiente de la Infancia Temprana, Edición Revisada (ECERs-R) de Harms, Clifford y Cryer (1998), y la Escala de Calificación del Ambiente para Bebés y Niños Pequeños, Edición Revisada (ITERS-R) de Harms, Cryer y Clifford (2003), son dos instrumentos ampliamente utilizados con el fin evaluar calidad de centros infantiles en diferentes países del mundo, dada su confiabilidad, validez y capacidad discriminatoria.

La ITERS-R ha sido diseñada para la evaluación y mejora de centros infantiles que atienden a niños entre cero y dos años y medio. Contempla 39 ítems organizados en siete subescalas: mobiliario y materiales; rutinas de cuidado personal; escuchar y hablar; actividades de aprendizaje; interacción; estructura del programa, y necesidades de los adultos. La consistencia interna de todas las escalas es 0,92 (Harms, Cryer y Clifford, 2003).

Por su parte, la escala ECERs-R, consta de 43 preguntas organizadas en siete escalas: espacio y 
muebles; rutinas de cuidado personal; lenguaje-razonamiento; actividades; interacción; estructura del programa; y padres y personal. Al igual que la ITERs, la consistencia interna de todas las escalas es 0,92 (Harms, Clifford y Cryer, 1998).

Según Lera-Rodríguez (2007) las escalas abarcan un modelo educativo estructurado en ciertos principios básicos, tales como la importancia de las interacciones que se establecen entre educadora y niño en situaciones informales; la calidad del espacio físico de la sala, el cual incluye materiales variados, mobiliario adecuado, organización y preocupación por la seguridad y confort de los niños; el desarrollo en áreas tales como lenguaje, motricidad, creatividad, habilidades sociales; y las necesidades de los adultos y padres.

Las orientaciones pedagógicas de estas escalas se pueden resumir así:

- Los niños aprenden con mayor facilidad, a través de actividades en las que experimentan y piensan por sí mismos, con la guía del adulto.

- Los niños aprenden de la interacción con sus padres, educadores u otros adultos, como también de la interacción con otros niños.

- Un entorno con espacio y materiales organizados, que permita a los niños ser independientes y aprovechar su tiempo en actividades significativas y productivas.

- Los niños requieren de un ambiente en el que se satisfagan sus necesidades de afecto, de seguridad y protección.

- Un entorno adecuado para los niños, debe también satisfacer las necesidades de los adultos que trabajan con ellos.

\section{El Classroom Assessment Scoring System (CLAss)}

El Sistema de Calificación para Evaluaciones en el Salón de Clases (CLASS), es un instrumento de observación desarrollado en la Universidad de Virginia a fin de evaluar la calidad en los salones de clases del nivel de educación parvularia. El mismo ha sido validado en más de 2000 salones de clases, y fue seleccionado por su confiabilidad para evaluar la calidad en los salones de clases de Head Start, en Estados Unidos. Cabe destacar que este instrumento también ha sido utilizado en la formación inicial docente.

CLASs evalúa la interacción entre niños-maestros en tres dominios de calidad en el salón de clases: apoyo emocional, organización en el aula, y apoyo didáctico. El apoyo emocional se refiere a conductas específicas de enseñanza que ayudan a los niños a desarrollar relaciones cálidas y solidarias, a sentir alegría y entusiasmo por aprender, a sentirse cómodos en el aula y a experimentar niveles apropiados de autonomía e independencia. Por su parte, la organización del aula describe las conductas específicas de enseñanza que ayudan a los niños a desarrollar habilidades para regular su propio comportamiento, aprovechar al máximo el aprendizaje de cada día y mantener el interés en las actividades de aprendizaje. Finalmente, el apoyo didáctico se refiere a conductas específicas de enseñanza que promueven el desarrollo cognitivo y lingüístico de los niños. Este instrumento ha sido utilizado en numerosos estudios y programas (Hamre et al., 2013), por ejemplo, en el programa Head Start implementado en Estados Unidos.

\section{El contexto chileno}

En Chile se entiende la educación parvularia como el primer nivel del sistema educacional chileno, reconocido en la Constitución Política de la República del año 1999, en virtud de la Ley 19634, Ley Orgánica Constitucional de Enseñanza (LOCE). Atiende integralmente a niños y niñas desde su nacimiento hasta su ingreso a la educación básica, sin constituirse como nivel obligatorio (MEN, 1997). Para el Ministerio de Educación (2001) este nivel educacional se propone favorecer en forma sistemática, oportuna y pertinente, aprendizajes de calidad para todos los niños y niñas menores de seis años, hasta su ingreso a la educación básica, a través de diversos organismos e instituciones y de forma complementaria a la educación que realizan las familias.

Esto desde la década de los noventa en la que se empezaron a implementar programas destinados a mejorar la calidad de la educación de los niños menores de seis años. Ejemplos de estos programas son Manolo y Margarita aprenden con sus padres, programa de articulación entre la educación parvularia y la educación general básica, Programa Conozca a su hijo, y programa de mejoramiento de la infancia (PMI). Por otra parte, en el 2001 se crean las bases curriculares de la educación parvularia (MEN, 2001), las cuales plantean una actualización de los fundamentos de este nivel, y constituyen la propuesta de un marco orientador para todos aquellos profesionales que se relacionan con los niños de esta edad. 
La educación parvularia es impartida tanto por organismos públicos como privados. En sectores de menores recursos, la mayor parte de la oferta proviene de sectores públicos, de manera que tres son los organismos responsables:

1. El Ministerio de Educación (MEN) a cargo principalmente del nivel transición (niños de cuatro a cinco años) de las escuelas municipales y subvencionadas.

2. La Junta Nacional de Jardines Infantiles (JUNJI), corporación de derecho público que se financia mediante el presupuesto fiscal anual y tiene como objetivo principal ofrecer educación integral gratuita a niños entre cero y cinco años, provenientes de familias de los sectores más vulnerables y de menores ingresos.

3. Finalmente, Fundación Integra es una institución de derecho privado, sin fines de lucro que, a partir de la década de los noventa, crea un proyecto educativo institucional dirigido principalmente por equipos técnicos.

El principal logro alcanzado durante estos años ha sido el aumento de la cobertura (por ejemplo, en los niños de cuatro años se evidenció un aumento del $35 \%$ en el 2003, y del $45 \%$ en el 2009), producto de la política implementada en los distintos gobiernos. Sin embargo, la tensión entre cobertura y calidad ya ha sido puesta en la discusión pública. La preocupación por la calidad de la educación inicial, particularmente en la instrucción en la sala y apoyo al lenguaje del niño (Herrera y Bellei, 2002; Eyzaguirre y Le Foulon, 2001; Strasser y Lissi, 2009; Villalón, Suzuki, Herrera y Mathiesen, 2002), es hoy un tema relevante.

Una investigación que evidencia algunas de las debilidades en el contexto chileno es el estudio de Treviño (2013). Esta investigación se centra en la descripción del desempeño de las educadoras de párvulos en una muestra de escuelas públicas que ofrecen pre-kínder a poblaciones vulnerables en las regiones Metropolitana y vi de Chile $(\mathrm{n}=118)$. Para tal efecto se usa la pauta de observación CLAss de pre-kinder (Treviño, 2013, p. 40). Los resultados indican que las prácticas en la muestra alcanzan niveles medios de desempeño en los dominios de apoyo emocional y organización de la clase, y niveles bajos en apoyo pedagógico. Por último, el desempeño de las educadoras en el aula es heterogéneo, logrando identificarse cuatro grupos distintos que requerirían programas de desarrollo profesional docente adaptados específicamente para mejorar en los ámbitos de menor desempeño en el aula. De acuerdo con este estudio, el conocimiento pedagógico del contenido permanece como una debilidad en el desempeño de los educadores, lo cual va en desmedro de una educación de calidad. Sin embargo, se observan iniciativas que buscan evaluar y mejorar esta situación. Ejemplo de ello es la formulación de los Estándares orientadores para carreras de educación parvularia (MEN, 2012), y la implementación de la prueba inicia para aquellos estudiantes que entran al mercado laboral.

\section{Algunas intervenciones que buscan el aumento de la calidad}

En los últimos años se han implementado en Chile una serie de programas e iniciativas que buscan mejorar la calidad de la educación parvularia, motivados por la evidencia que respalda la importancia e impacto de los contextos vitales en esta etapa.

Estas intervenciones surgen de diversos sectores (Estado, organizaciones no gubernamentales -ONG-, fundaciones). A continuación se presentan dos ejemplos de estas iniciativas y las evaluaciones que surgen desde ellas.

\section{Un buen comienzo}

El programa Un buen comienzo (UBC) diseñado por la Universidad de Harvard, el Ministerio de Educación y una mesa técnica intersectorial, e implementado por la Fundación Oportunidad en Chile, se trata de un programa diseñado en principio por la Universidad de Harvard (Mena, Lissi, Alcalay y Milicic, 2013), y el cual tiene como objetivos:

- Reducir los niveles de dificultad lectora que presentan niños/as de familias que viven en situación de vulnerabilidad social en Chile.

- Reducir la brecha en el vocabulario existente entre niños de familias de bajos ingresos y sus pares más privilegiados.

- Promover un ambiente positivo en la sala de clase que permita el aprendizaje de los niños/as.

- Mejorar la asistencia a la educación parvularia adoptando medidas de salud preventivas, especialmente en el área de las enfermedades respiratorias, mediante los chequeos de salud y los controles de niño sano.

- Promover la participación de los padres en la educación inicial de sus niños/as. 
Este proyecto se diseña a partir de la preocupación por mejorar la calidad de la educación parvularia en Chile. Se trata de una intervención de desarrollo profesional docente. Es implementado y evaluado en seis comunas vulnerables de la región metropolitana en Chile. En su conjunto, es financiado e implementado por la fundación educacional Oportunidad.

Se llevan a cabo doce ciclos (véase figura 2) en dos años de intervención. Este acompañamiento comprende una etapa "pre", un acompañamiento propiamente tal y una etapa "post".

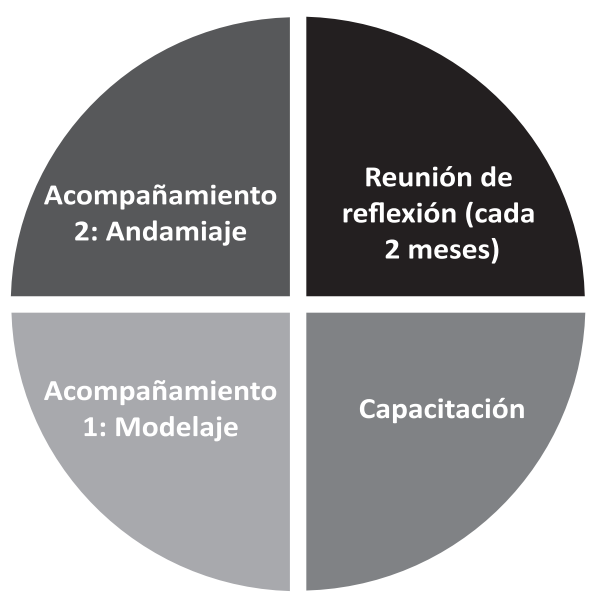

Figura 2. Ciclo de implementación del programa Un buen comienzo. Tomado de Yoshikawa, H. (2012, abril). Creando Oportunidad 2. Seminario Internacional de Educación Inicial. Seminario llevado a cabo en Santiago de Chile.

Cabe destacar en el ciclo de implementación del programa, el acompañamiento que se les brinda a los educadores: capacitación, se modela la práctica, construcción de las preguntas, y reflexión en conjunto con ellos a partir de su contexto determinado. Esta iniciativa es interesante considerando que, en general, los proyectos comprenden una intervención puntual y una evaluación de ella. Esta característica del programa UBC de acompañar y reflexionar junto con los involucrados, es sin duda un factor que ayudará a la sustentabilidad del cambio.

Los resultados mostrados por Hirokazu Yoshikawa en la Presentación Seminario Internacional de Educación Inicial 2, organizado por la Fundación Oportunidad (ver figura 3), mostró un gran aumento en el apoyo emocional (actividades compartidas, emociones y expectativas positivas, interacciones positivas y respetuosas); un aumento moderado en el apoyo instruccional (preguntas y enunciados abiertos, disposición, resolución de problemas, predicción, clasificación, lluvia de ideas, expansión, elaboración, clarificación, planificación, intercambios mutuos); y un aumento moderado en la organización de la sala (actividades de aprendizaje con pocas interrupciones, instrucciones claras, transiciones breves, preparación de la clase y el material).

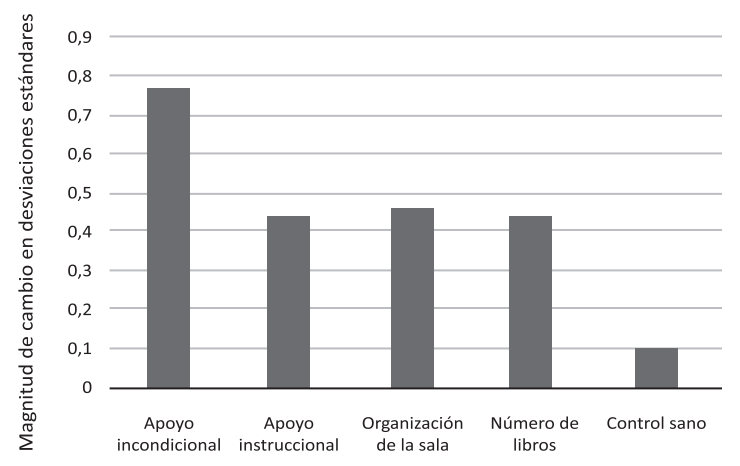

Figura 3. Tamaño del efecto del impacto en calidad de la sala de clases. Tomado de Yoshikawa, H. (2012, abril). Creando Oportunidad 2. Seminario Internacional de Educación Inicial. Seminario llevado a cabo en Santiago de Chile.

El programa Un buen comienzo ha seguido desarrollándose y queda un registro de sus avances y resultados en diversos artículos (Yoshikawa et al., 2015; Leyva et al., 2015; Leyva y Nolivos, 2015).

\section{Implementación del programa Quik en Colina}

La implementación del programa Quik se llevó a cabo en un sector de escasos recursos de la comuna de Colina de Santiago. Se trata de una propuesta desarrollada por Wolfgang Tietze y su equipo en Alemania, la cual fue adaptada en Chile por M. Olivia Herrera (Tietze \& Viernickel, 2010) en sus libros Desarrollo de la calidad educativa en centros prescolares, y Manual de desarrollo de la calidad educativa.

La corporación People Help People, encargó a un equipo de docentes de la Facultad de Educación, liderado por Cynthia Adlerstein, la evaluación de la propuesta de aseguramiento de la calidad del programa Quik con el fin de analizar su capacidad de impactar 
los ambientes de aprendizaje, dentro de diecinueve centros de educación inicial en la Municipalidad de Colina. Para esto se utilizó un modelo de investigación mixto al aplicar las escalas de evaluación ECERs e ITERS, y llevar a cabo entrevistas y grupos focales con educadores, técnicos, directoras y apoderados. Los resultados de la evaluación de las escalas se presentan en la figura 4.

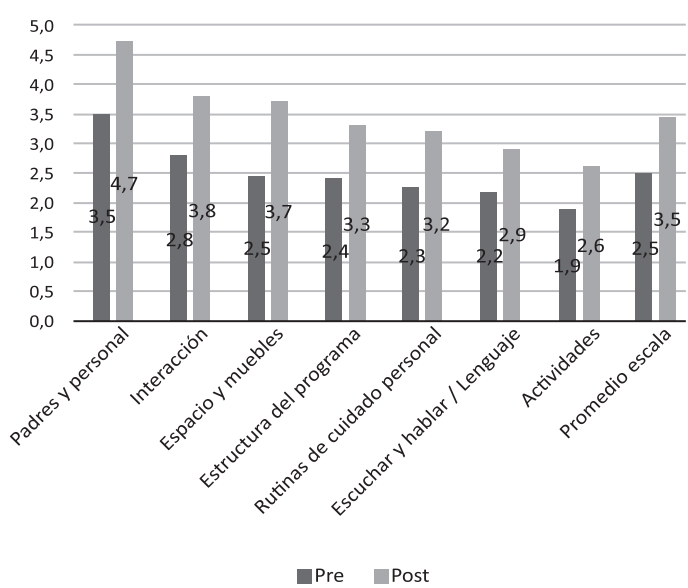

Figura 4. Promedios ECERS-R / ITERS-R pre y post aplicación Quik por subescala. Tomado de Adlerstein, C., Diaz, C., Suzuki, E. y Valverde, P. (2012). Evaluación de la implementación y resultados del programa Quik en seis centros de educación parvularia de la municipalidad de Colina (Presentación Informe Final, inédito). Chile: Pontificia Universidad Católica de Chile.

Los resultados muestran un efecto positivo en todos los jardines y claramente el programa logra un efecto en ellos. Interesante destacar que hay dimensiones que suben más que otras: padres y personal, y espacio y muebles (se observa un aumento de 1,2 puntos en ambos ítems).

Sin embargo, a pesar del aumento observado, las mediciones develan aún cifras poco óptimas: el logro de las caracterizaciones hechas con estos instrumentos en nuestro país llegan al punto 3, lo que es bajo si se observa que el puntaje máximo es 7. Claramente y a pesar de los esfuerzos realizados hasta el momento, aún hay grandes desafíos por delante, cuando se trata del tema de la calidad en educación parvularia.

\section{Reflexiones finales}

Una vez revisados algunos ejemplos de programas y evaluaciones de calidad en Chile, es evidente que el tema es de alta relevancia y sin duda uno de los ejes de preocupación y discusión en la agenda pública.

Es en estas discusiones en las que se pueden identificar algunas tensiones. La primera tensión es entre la educación inicial entendida como servicio de carácter asistencial versus la educación inicial entendida como un servicio de carácter educativo. En la primera se asegura el "cuidado" de los niños, por lo que la calidad estaría centrada en aspectos ligados más a la seguridad, a la alimentación y a la salud. En la segunda mirada, el foco estaría centrado en el aprendizaje y, por lo tanto, las variables ligadas al ambiente revisadas en este escrito revelarían, entre otras, caminos para buscar el mejoramiento de la calidad. Esta tensión entre estos focos tiene sus raíces en las motivaciones históricas que estaban a la base de la creación de este nivel educativo.

Una segunda tensión se da respecto a cuál es el actor focalizado para buscar la calidad: en este caso, el adulto o el niño. Esta tensión impacta el proceso educativo desde su planificación hasta su evaluación, y puede ser entendida también desde la tensión entre dos conceptos: el enseñar y el aprender.

Otra tensión que podemos identificar y es la más claramente presente en la discusión pública de nuestro país, se da entre calidad y cobertura. Respecto a esto, Chile ha alcanzado un buen nivel de cobertura (en el 2011 la educación parvularia cubría a cerca de 700000 niños matriculados, lo que alcanzaba a un $44 \%$ de los niños entre cero y cinco años) ${ }^{1}$,pero también se han diagnosticado factores culturales que condicionan la asistencia afectando en ello variables como la edad del niño y el trabajo de las madres. Recientes estudios han tenido como propósito evaluar el impacto de la participación de los niños en este nivel en el resultado de pruebas estandarizadas que se aplican en la educación básica (SIMCE). Los resultados generales relevan el positivo impacto que tiene asistir a la educación parvularia, así como la importancia de que esta sea de calidad. Sin embargo, cabe destacar que los resultados especifican que independientemente de la metodología utilizada, la asistencia a los primeros niveles tiene un efecto promedio "negativo sobre el puntaje SIMCE" (MEN, 2014).

\footnotetext{
1 Datos de la encuesta sobre Caracterización Socioeconómica Nacional - CASEN, 2011, en MEN (2012)
} 
Junto con la complejidad de estas tensiones, esto se enmarca en un contexto en el que la atención de los niños en jardines infantiles ha sido objeto de denuncia pública por maltrato y abuso. Estas denuncias han debilitado la confianza de los padres en los centros educativos, lo que complejiza la relación con las familias impactando también la cualidad de la interacción del educador con los niños que tiene a su cargo.

Estas tensiones y características de este contexto reflejan algunos de los desafíos que enfrenta Chile como país en este camino de búsqueda del mejoramiento de los ambientes de calidad en la infancia temprana.

\section{Referencias}

Adlerstein, C., Diaz, C., Suzuki, E. y Valverde, P. (2012). Evaluación de la implementación y resultados del programa Quik en seis centros de educación parvularia de la Municipalidad de Colina. (Presentación Informe Final, inédito). Chile: Pontificia Universidad Católica de Chile.

Aguerrondo, I. (1993). La calidad en educación: Ejes para su definición y evaluación. La Educación, 37(116), 561578 .

Copple, C. y Bredekamp, S. (Eds.). (2009). Developmentally appropriate practice in early childhood programs: serving children from birth through age 8 . ( $3^{\mathrm{a}}$ ed.). Washington, DC: NAEYC.

Clark-Stewart, A. (1993). Daycare. Cambridge: Harvard University Press.

Delors, J. (1996). La educación encierra un tesoro. Informe de la UNESCO. Madrid: Santillana.

De Vries, R. y Zan, B. (2003). When Children Make Rules Educational Leadership, 61(1), 64-67.

Dombro, A., Jablon, J. y Stetson, C. (2011). Powerful Interactions. Young Children, 66(1), 12-20.

Egido, I. (2000). La educación inicial en el ámbito internacional: Situación y perspectivas en Iberoamérica y en Europa. Revista Iberoamericana de Educación, 22, 119-154.

Eyzaguirre, B. y Le Foulon, C. (2001). La calidad de la educación chilena en cifras. Estudios Públicos, 84, 85-204. Recuperado de: http://ww2.educarchile.cl/ 55985AED-ECCC-48D4-A631-54465A6EBC24/FinalDownload/ DownloadId295B08B2CF0D66BDAE1FBFE77C9E066A/55985AED-ECCC-48D4-A63154465A6EBC24/UserFiles/P0001/File/Calidad\%20 ed.\%20en\%20cifrasEyzaguirre.pdf
Goleman, H. y Pence, A. R. (1987). Effects of child care family or individual characteristics on children's language development: The Victoria Day care Research. En D. A. Philips (Ed.), Quality and child care: What does research tell us? (pp. 89-104). Washington, DC: National Association for the Education of Young Children.

Hadred, J. y Sylva, K. (1994). The effects of care and education oriented pre-school experience on childrens development in Bahrain. Gothenburg, Sweden: Fourth European Early Childhood Education Research Association Conference on the Quality of Early Childhood Education.

Hamre, B., Pianta, R., Downer, J. y DeCoster, J. (2013). Teaching through Interactions Testing a Developmental Framework of Teacher Effectiveness in over 4,000 Classrooms. The Elementary School Journal, 113(4), 461-487. doi: http://dx.doi.org/10.1086/669616

Harms, T., Clifford, R. y Cryer, D. (1998). Early Childhood Environment Rating Scale (Rev. ed.). New York: Teachers College Press.

Harms, T., Cryer, D. y Clifford, R. M. (2003). Infant/Toddler Environment Rating Scale, Revised Edition. New York: Teachers College Press.

Herrera. M. O. y Bellei, C. (2002). ¿Qué se sabe sobre la calidad de la educación parvularia chilena? Santiago de Chile: Universidad de Concepción y UnICEF.

Howes, C. (1988). Relations between Child Care and Schooling. Developmental Psychology, 24, 53-57. doi: 10.1037/0012-1649.24.1.53

Kontos, S. y Fienes, R. (1987). Predictors of quality and children's development in day care. En D. Phillips (Ed.), QuaIity indicators of child care (pp. 57-79). Washington, DC: National Association for the Education of Young Children.

Lavanchy, S. (1993). Educación preescolar: Desafío y aventura. Santiago: Editorial Universitaria.

Lera-Rodríguez, M. J. (2007). Calidad de la Educación infantil: instrumentos de evaluación. Revista de Educación, 343, 301-323.

Leyva y Nolivos, V. (2015). Chilean Family Reminiscing About Emotions and Its Relation to Children's Self-Regulation Skills. Early Education and Development, 25(5-6), 770-791. doi: http://dx.doi.org/10.1080 /10409289.2015.1037625

Leyva, D., Weiland, C., Barata, M., Yoshikawa, H., Snow, C., Treviño, E. y Rolla, A. (2015), Teacher-Child Interactions in Chile and Their Associations With Prekindergarten Outcomes. Child Development, 86, 781-799. 
Disponible en http://www.fundacionoportunidad.cl/ assets/uploads/archivos/e885a-classchile_2015_leyva_weiland-1.pdf

Malaguzzi, L. (1996). Los cien lenguajes de los niños. Nidos y Escuelas de la Infancia, Instituciones de la Municipalidad de Reggio Emilia. Reggio Emilia: Reggio Children.

Meece, D. y Soderman, A. (2010). Positive Verbal Environments. Setting the Stage for Young Children's Social Development. Young Children, 65(5), 81-86. Recuperado de: http://eric.ed.gov/?id=EJ898737

Mena, I., Lissi, M. R., Alcalay, L. y Milicic, N. (Eds.). (2013). Educación y diversidad. Santiago: Ediciones UC.

Melhuish, E. (1993). Preeschool care and education: lessons from the 20th for the 21st Century. International journal of early years education, 1(2), 19-32. doi:10.1080/0966976930010203

Ministerio de Educación. [MEN]. (1997). Ley Orgánica Constitucional de Enseñanza (LOCE). Santiago de Chile: Gobierno de Chile.

Ministerio de Educación. [MEN]. (2001). Bases Curriculares de la Educación Parvularia. Unidad de Curriculum y Evaluación. Santiago de Chile: Ministerio de Educación. Gobierno de Chile.

Ministerio de Educación. [MEN]. (2012a). Encuesta CASEN 2011, Análisis Módulo Educación. Recuperado de static1.mineduc.cl/doc-mailing/20121026\%20analisis\%20casen\%20final.pdf

Ministerio de Educación. [MEN]. (2012b). Estándares orientadores para carreras de educación parvularia. Santiago de Chile.

Ministerio de Educación. [MEN]. (2014). Nueva evidencia sobre el impacto de la educación parvularia. Recuperado de http://static.mineduc.cl/doc_mailing/evidencias_cem_26.pdf

National Scientific Council on the Developing Child. (2004). Young children develop in an environment of relationships. Working Paper N. ${ }^{\circ}$ 1. Recuperado de: http:// www.developingchild.net

NAEYC. (2009). Declaración de Posición de NAEYC. Recuperado de https://www.naeyc.org/files/naeyc/file/positions/Spanish \%20DAP\%20position\%20statement\%20(1).pdf
Phillips, D. y Howes, C. (1987). Indicators of quality in child care: Review of the research. En D. A. Phillips (Ed.), Quality in child care: What does research tell us? (pp. 1-19). Washington, DC: National Association for the Education of Young Children.

Phillipsen, L. C., Burchinal, M. R., Howes, C. y Cryer, D. (1997). The prediction of process quality from structural features of child care. Early Childhood Research Quarterly, 12 281-303. doi: 10.1016/S08852006(97)90004-1

Pianta, R., La Paro, K. M. y Hamre, B. K. (2012). Classroom Assessment Scoring System (CLASS). Manual, pre-K. Baltimore: Paul H. Brookes Publishing.

Strasser, K. y Lissi, M. R. (2009). Home and Instruction Effects on Emergent Literacy in a Sample of Chilean Kindergarten Children. Scientific Studies of Reading, 13(2), 175-204. doi: 10.1080/10888430902769525

Tietze, W. y Viernickel, S. (2010). Desarrollo de la calidad educativa en centros preescolares (G. Castro y M. O. Herrera, Trad.). Santiago de Chile: Lom.

Treviño, E. (2013). Calidad de la educación parvularia: las prácticas de clase y el camino a la mejora. Pensamiento educativo. Revista de Investigación Educacional Latinoamericana, 5, 40-62. doi:10.7764/PEL.50.1.2013.4

Villalón, M., Suzuki, E., Herrera, M. O. y Mathiesen, M. E. (2002). Quality of Chilean Early Childhood Education from an International Perspective. International Journal of Early Years Education, 10(1), 49-59. doi: http:// dx.doi.org/10.1080/09669760220114845

Yoshikawa, H. (2012, abril). Creando Oportunidad 2. Seminario Internacional de Educación Inicial. Seminario llevado a cabo en Santiago de Chile.

Yoshikawa, H., Leyva, D., Snow, C., Treviño, E., Barata, C., Wellando, C., ... Arbour, M. C. (2015). Experimental impacts of a teacher professional development program in Chile on preschool classroom quality and child outcomes. Developmental Psychology, 51(3), 309322. doi: http://dx.doi.org/10.1037/a0038785 